\title{
Evaluation of Generalized Howland Integrals
}

\section{By Chih-Bing Ling}

\begin{abstract}
This paper presents a method of evaluation of the generalized Howland integrals
\end{abstract} The values are tabulated to 10D.

The generalized Howland integrals are defined by

$$
\begin{aligned}
& I_{k, s}=\frac{2^{k}}{k !} \int_{0}^{\infty} \frac{x^{k} e^{-s x} d x}{\sinh 2 x \pm 2 x}=\frac{1}{2(k !)} \int_{0}^{\infty} \frac{x^{k} e^{-s x / 2} d x}{\sinh x \pm x}, \quad(k \geqslant 1) \\
& (k \geqslant 3)
\end{aligned}
$$

where $k$ and $s$ are integers. For the sake of convergence, $k$ is restricted as indicated above and $s$ is restricted to be not less than -1 . Owing to their frequent occurrence in mathematical sciences, it is thought that they deserve a special consideration.

The four integrals for $s=0$ and 2 are the ordinary Howland integrals. The two integrals for $s=0$ have been evaluated to 25D by Ling and Lin [3] when $k$ is odd and by Ling [4] when $k$ is even. Those for $s=2$ have recently been evaluated to 20D by Ling and $\mathrm{Wu}[5]$. It is the endeavor of the present paper to evaluate the remaining integrals to $10 \mathrm{D}$.

The following recurrence relations for $s \geqslant 1$ are readily verified:

$$
\begin{aligned}
& I_{k, s-2}+2(k+1) I_{k+1, s}-I_{k, s+2}=\left(\frac{2}{s}\right)^{k+1}, \\
& I_{k, s-2}^{*}-2(k+1) I_{k+1, s}^{*}-I_{k, s+2}^{*}=\left(\frac{2}{s}\right)^{k+1} .
\end{aligned}
$$

By using these relations, the integrals $I_{k+1, s}$ and $I_{k+1, s}^{*}$ can be evaluated by recurrence in terms of $I_{k, s-2}, I_{k, s+2}$ and $I_{k, s-2}^{*}, I_{k, s+2}^{*}$ from the values of the leading integrals $I_{k,-1}, I_{k, 0}, I_{1, s}$ and $I_{k,-1}^{*}, I_{k, 0}^{*}, I_{3, s}^{*}$, respectively. Such a process of computation has the distinct advantage that no accuracy is lost in successive steps, except perhaps when $s=1$. To avoid this possibility, we take $I_{k, 1}$ and $I_{k, 1}^{*}$ as the leading integrals intead of $I_{k,-1}$ and $I_{k,-1}^{*}$.

As mentioned before, the integrals $I_{k, 0}$ and $I_{k, 0}^{*}$ have been evaluated to high precision of 25D. Plana's method was used for their evaluation. This method, however, is no longer applicable if the value of $s$ in the integrals is other than zero. By expanding $e^{-x / 2}$ in the second form of the integrands in (1) into a series in $x$,

Received March 24, 1981; revised July 7, 1981

1980 Mathematics Subject Classification. Primary 65A05, 65D20, 65D30.

Key words and phrases. Howland integrals, generalized Howland integrals. 
integrating and then applying the Kummer transformation [1], the following relations are found for $s \geqslant 1$ :

$$
\begin{aligned}
& \left(\frac{2}{s+2}\right)^{k+1}-I_{k, s}=\sum_{n=0}^{\infty} \frac{(-1)^{n}}{2^{n}}\left(\begin{array}{c}
n+k \\
n
\end{array}\right)\left\{\left(\frac{2}{s+2}\right)^{n+k+1}-I_{n+k, s-1}\right\}, \\
& I_{k, s}^{*}-\left(\frac{2}{s+2}\right)^{k+1}=\sum_{n=0}^{\infty} \frac{(-1)^{n}}{2^{n}}\left(\begin{array}{c}
n+k \\
n
\end{array}\right)\left\{I_{n+k, s-1}^{*}-\left(\frac{2}{s+2}\right)^{n+k+1}\right\} .
\end{aligned}
$$

Here the ratio of the binomial coefficient to $2^{n}$ may or may not be greater than unity. When it is, a certain amount of accuracy is lost. The loss is larger, when $k$ is larger. For instance, the loss is $5 \mathrm{D}$ when $k=20$ and $10 \mathrm{D}$ when $k=36$.

It appears possible to reduce the loss of accuracy if the computation for a unit increment of $s$ is carried out in several steps instead of a single step as in (3). Suppose that four steps are taken such that in each step the increment of $s$ is $\frac{1}{4}$. Then, in the $r$ th step, for $r=1,2,3$, or 4 , the intermediate integrals are given by

(4)

$$
\begin{aligned}
& \left(\frac{8}{4 s+r+8}\right)^{k+1}-I_{k, s+\frac{1}{4} r} \\
& \quad=\sum_{n=0}^{\infty} \frac{(-1)^{n}}{8^{n}}\left(\begin{array}{c}
n+k \\
n
\end{array}\right)\left\{\left(\frac{8}{4 s+r+7}\right)^{n+k+1}-I_{n+k, s+\frac{1}{4} r-\frac{1}{4}}\right\}, \\
& I_{k, s+\frac{1}{4} r}^{*}-\left(\frac{8}{4 s+r+8}\right)^{k+1} \\
& \quad=\sum_{n=0}^{\infty} \frac{(-1)^{n}}{8^{n}}\left(\begin{array}{c}
n+k \\
n
\end{array}\right)\left\{I_{n+k, s+\frac{1}{4} r-\frac{1}{4}}^{*}-\left(\frac{8}{4 s+r+7}\right)^{n+k+1}\right\} .
\end{aligned}
$$

It is seen that the binomial coefficient involved is now divided by $8^{n}$ instead of $2^{n}$. When $k=18$, the accumulated loss of accuracy in four steps together is reduced to slightly less than $2 \mathrm{D}$ only. Hence, if we begin the computation with the $25 \mathrm{D}$ values of $I_{k, 0}$ and $I_{k, 0}^{*}$ and take four steps for each unit increment of $s$, we can find up to $k=18,23 \mathrm{D}$ values of $I_{k, 1}$ and $I_{k, 1}^{*}, 21 \mathrm{D}$ values of $I_{k, 2}$ and $I_{k, 2}^{*}$, and 19D values of $I_{k, 3}$ and $I_{k, 3}^{*}$, successively. However, values of the intermediate integrals at each step for $k \geqslant 19$ are also needed in the computation. These values can be found directly by developing the integrals into series [5] as follows:

$$
\begin{aligned}
& I_{k, s+r / 4} \\
& I_{k, s+r / 4}^{*}
\end{aligned}=\sum_{n=1}^{\infty}(\mp 1)^{n+1} q_{n}\left(k, s+\frac{r}{4}\right)\left(\frac{8}{8 n+4 s+r}\right)^{k+1},
$$

where, for $n \geqslant 0$,

(6)

$$
q_{2 n+1}\left(k, s+\frac{r}{4}\right)=\sum_{t=0}^{\infty}\left(\begin{array}{c}
k+2 t \\
k
\end{array}\right) \frac{(n+t) !}{(n-t) !}\left(\frac{16}{16 n+4 s+r+8}\right)^{2 t},
$$

$$
q_{2 n+2}\left(k, s+\frac{r}{4}\right)=\sum_{t=0}^{\infty}\left(\begin{array}{c}
k+2 t+1 \\
k
\end{array}\right) \frac{(n+t+1) !}{(n-t) !}\left(\frac{16}{16 n+4 s+r+16}\right)^{2 t+1} .
$$

When $k=19$, the series in (5) are to be carried to $n=190$ for 23D, to $n=100$ for 21D, and to $n=50$ for 19D. For 10D, the corresponding value of $n$ is 14 when $k=15$, or 6 when $k=19$. The convergence of the series increases with $k$ but only slightly with $s$. 
When the values of the integrals for $s=0,1,2$, and 3 are available in high precision as described above, we can use the recurrence relations in (2) to compute $I_{k, s+2}$ and $I_{k, s+2}^{*}$ for $s \geqslant 2$ in terms of $I_{k, s-2}, I_{k+1, s}$ and $I_{k, s-2}^{*}, I_{k+1, s}^{*}$, respectively. Owing to the factor $2(k+1)$ associated with $I_{k+1, s}$ and $I_{k+1, s}^{*}$, some accuracy is always lost. The loss is 1D when $k=4,1 \frac{1}{2} \mathrm{D}$ when $k=14$, or $2 \mathrm{D}$ when $k=49$. Hence the integrals can be computed successively so long as the desired accuracy of 10D is still sustained. In this manner, values of a considerable number of integrals are obtained, including the leading integrals $I_{1, s}$ and $I_{3, s}^{*}$. There is ground to claim that the values of $I_{1, s}$ and $I_{3, s}^{*}$ thus obtained up to $s=20$ and 18, respectively, are accurate to $10 \mathrm{D}$.

For further evaluation of $I_{1, s}$ and $I_{3, s}^{*}$, consider the asymptotic expansion of these integrals. We begin by changing the variable $x$ in the integrals with the substitution

$$
e^{x}=1+y=1+x+\frac{x^{2}}{2 !}+\frac{x^{3}}{3 !}+\cdots
$$

Reversion of the series yields

$$
x=\ln (1+y)=y-\frac{y^{2}}{2}+\frac{y^{3}}{3}-\frac{y^{4}}{4}+\cdots .
$$

The powers of $x$ can therefore be expressed as series in $y$. By differentiation, we also find

$$
d x=d y /(1+y)
$$

Next, find the reciprocals of the following expansions of $x$ :

$$
\begin{aligned}
& \frac{\sinh x+x}{x}=2+\frac{x^{2}}{3 !}+\frac{x^{4}}{5 !}+\frac{x^{6}}{7 !}+\cdots \\
& \frac{\sinh x-x}{x^{3}}=\frac{1}{3 !}+\frac{x^{2}}{5 !}+\frac{x^{4}}{7 !}+\frac{x^{6}}{9 !}+\cdots
\end{aligned}
$$

We have

$$
\begin{aligned}
\frac{2 x}{\sinh x+x} & =1-\frac{x^{2}}{12}+\frac{x^{4}}{360}+\frac{x^{6}}{60,480}-\frac{11 x^{8}}{1,814,400}+\cdots, \\
\frac{x^{3}}{6(\sinh x-x)} & =1-\frac{x^{2}}{20}+\frac{11 x^{4}}{8400}-\frac{17 x^{6}}{756,000}+\frac{563 x^{8}}{2,328,480,000}-\cdots .
\end{aligned}
$$

Suppose that these series in $x$ are expressed as series in $y$ in the form:

$$
\frac{2 x}{\sinh x+x}=1+\sum_{m=2}^{\infty} p_{m} y^{m}, \quad \frac{x^{3}}{6(\sinh x-x)}=1+\sum_{m=2}^{\infty} p_{m}^{*} y^{m} \text {. }
$$

The following values are found:

\begin{tabular}{c|ccccccc}
$m$ & 2 & 3 & 4 & 5 & 6 & 7 & 8 \\
\hline$p_{m}$ & $-\frac{1}{12}$ & $\frac{1}{12}$ & $-\frac{53}{720}$ & $\frac{23}{360}$ & $-\frac{3359}{60,480}$ & $\frac{979}{20,160}$ & $-\frac{155,083}{3,628,800}$ \\
$p_{m}^{*}$ & $-\frac{1}{20}$ & $\frac{1}{20}$ & $-\frac{187}{4200}$ & $\frac{41}{1050}$ & $-\frac{12,991}{378,000}$ & $\frac{3841}{126,000}$ & $-\frac{881,701}{32,340,000}$
\end{tabular}

To proceed further, let

$$
y=\sin ^{2} \theta / \cos ^{2} \theta
$$


Consequently,

$$
e^{-x / 2}=\cos \theta, \quad d x=2(\sin \theta / \cos \theta) d \theta
$$

We then have

$$
I_{1, s}=\frac{1}{2 s}+\frac{1}{4} \sum_{m=2}^{\infty} p_{m} H_{s, m}, \quad I_{3, s}^{*}=\frac{1}{s}+\frac{1}{2} \sum_{m=2}^{\infty} p_{m}^{*} H_{s, m},
$$

where

$$
\begin{aligned}
H_{s, m} & =2 \int_{0}^{\pi / 2}\left(\frac{\sin \theta}{\cos \theta}\right)^{2 m+1} \cos ^{s} \theta d \theta \\
& =m ! \Gamma\left(\frac{s}{2}-m\right) / \Gamma\left(\frac{s}{2}+1\right) \\
& =m ! /\left\{\frac{s}{2}\left(\frac{s}{2}-1\right)\left(\frac{s}{2}-2\right) \cdots\left(\frac{s}{2}-m\right)\right\} .
\end{aligned}
$$

By expanding the preceding expression into inverse power series of $s$, the following asymptotic series are obtained:

$$
\begin{aligned}
& I_{1, s} \sim \frac{1}{4}\left(\frac{2}{s}\right)-\frac{1}{24}\left(\frac{2}{s}\right)^{3}+\frac{1}{60}\left(\frac{2}{s}\right)^{5}+\frac{1}{336}\left(\frac{2}{s}\right)^{7}-\frac{11}{180}\left(\frac{2}{s}\right)^{9}+\cdots \\
& I_{3, s}^{*} \sim \frac{1}{2}\left(\frac{2}{s}\right)-\frac{1}{20}\left(\frac{2}{s}\right)^{3}+\frac{11}{700}\left(\frac{2}{s}\right)^{5}-\frac{17}{2100}\left(\frac{2}{s}\right)^{7}+\frac{563}{115,500}\left(\frac{2}{s}\right)^{9}-\cdots .
\end{aligned}
$$

The first series gives values to $10 \mathrm{D}, 11 \mathrm{D}, 12 \mathrm{D}$, when $s=16,20,24$, respectively, and the second when $s=10,13,16$, respectively.

As described before, 10D values of $I_{1, s}$ and $I_{3, s}^{*}$ have been found up to $s=20$ and 18 , respectively. It is thus seen that further $10 \mathrm{D}$ values of these two integrals can be found from the asymptotic series in (18) alone.

Lastly, to evaluate the remaining integrals $I_{k,-1}$ and $I_{k,-1}^{*}$, the following series may be used:

$$
\begin{aligned}
& \frac{1}{2^{k+1}} I_{k,-1}=1-\sum_{n=0}^{\infty} \frac{1}{2^{n+k+1}}\left(\begin{array}{c}
n+k \\
n
\end{array}\right)\left(1-I_{n+k, 0}\right) \\
& \frac{1}{2^{k+1}} I_{k,-1}^{*}=1+\sum_{n=0}^{\infty} \frac{1}{2^{n+k+1}}\left(\begin{array}{c}
n+k \\
n
\end{array}\right)\left(I_{n+k, 0}^{*}-1\right)
\end{aligned}
$$

which are found similarly by expanding $e^{x / 2}$ in (1) into a series in $x$. No accuracy is lost in this case since here the ratio of the binomial coefficient to $2^{n+k+1}$ is always less than unity.

The foregoing computation was carried out on an IBM 3032 computer with extended precision. In the course of computation, the values are generally carried with an accuracy exceeding 10D. Ample guard digits were provided whenever needed to given an extra accuracy as far as practicable. In several instances, the integrals were computed by different methods for some overlapping $k$ or $s$ to serve as a check. Finally, the results were rounded off to 10D and shown in Tables 1-4. For the sake of brevity, other values are not shown. Further $10 \mathrm{D}$ values of $I_{1, s}$ and $I_{3, s}^{*}$ in Table 1 are both given by the first three terms of the respective asymptotic 
series in (18). It may be mentioned that the first two terms of the series in (5) when $r=0$ are

$$
I_{k, s}^{*} \sim \frac{2^{k+1}}{(s+2)^{k+1}} \mp \frac{(k+1) 2^{k+3}}{(s+4)^{k+2}} .
$$

They give good approximation when $k$ is large. Further 10D values of $I_{k, 1}$ and $I_{k, 1}^{*}$ in Table 2 are given when $s=1$ by the first term only. If both terms are used, they give $10 \mathrm{D}$ values from $k=23$ onward.

TABLES $1 \& 2$

Values of $I_{1, s}, I_{3, s}^{*}$ and $I_{k, 1}, I_{k, 1}^{*}$

\begin{tabular}{|c|c|c|c|c|c|c|}
\hline $\begin{array}{r}\mathbf{s} \\
\text { or } \mathbf{k} \\
\end{array}$ & &,$s$ & $I_{3}^{*}, s$ & $\mathrm{I}_{\mathrm{k}, 1}$ & $I_{k, 1}^{*}$ & \\
\hline 1 & 0.35726 & 51300 & 0.7902190430 & 0.3572651300 & - & \\
\hline 2 & 0.22011 & 95814 & 0.4607137190 & 0.2108986635 & - & \\
\hline 3 & 0.15623 & 63163 & 0.3202175927 & 0.1460574537 & 0.79021 & 90430 \\
\hline 4 & 0.12028 & 34787 & 0.2441864095 & 0.1038005261 & 0.26920 & 28971 \\
\hline 5 & 0.09749 & 98574 & 0.1969488557 & $0.07348 \quad 74141$ & 0.13201 & 49095 \\
\hline 6 & 0.08185 & 80268 & 0.1648760199 & 0.0514600004 & 0.07467 & 03132 \\
\hline 7 & 0.07048 & 83836 & 0.1417196838 & 0.0356253748 & 0.04529 & 37207 \\
\hline 8 & 0.06186 & 52335 & 0.1242336202 & 0.0244208531 & 0.02853 & 83900 \\
\hline 9 & 0.05510 & 73534 & 0.1105707199 & 0.0166093689 & 0.01837 & 93682 \\
\hline 10 & 0.04967 & 20114 & $0.09960 \quad 49274$ & 0.0112295814 & 0.01199 & 26267 \\
\hline 11 & 0.04520 & 74267 & $0.09061 \quad 163$ & 0755933 & .00788 & 81283 \\
\hline 12 & 0.04147 & 59140 & 0.0831038443 & 0.0050728893 & 0.00521 & 42567 \\
\hline 13 & 0.03831 & 12562 & 0.0767423485 & 0.0033969198 & 0.00345 & 75221 \\
\hline 14 & 0.03559 & 38024 & $0.07128 \quad 37241$ & $0.00227 \quad 12707$ & 0.00229 & 71652 \\
\hline 15 & 0.03323 & 52717 & 0.0665488043 & 0.0015171047 & 0.00152 & 81325 \\
\hline 16 & 0.03116 & 91294 & 0.0624028195 & 0.0010126760 & 0.00101 & 73574 \\
\hline 17 & 0.02934 & 42938 & 0.0587424644 & 0.0006756671 & 0.00067 & 76484 \\
\hline 18 & 0.02772 & 09046 & 0.0554872329 & 0.0004506807 & 0.00045 & 15169 \\
\hline 19 & 0.02626 & 74072 & 0.0525734633 & 0.0003005545 & 0.00030 & 09065 \\
\hline 20 & 0.02495 & 85002 & 0.0499501563 & 0.0002004124 & 0.00020 & 05603 \\
\hline 21 & 0.02377 & 36613 & 0.04757597 .83 & 0.0001336264 & 0013 & 36883 \\
\hline 22 & 0.02269 & 60716 & 0.0454170769 & 0.0000890919 & 0.00008 & 91178 \\
\hline 23 & 0.02171 & 18169 & 0.0434454629 & 0.0000593978 & 0.00005 & 94086 \\
\hline 24 & 0.02080 & 92877 & 0.0416377944 & 0.0000395999 & 0.00003 & 96044 \\
\hline 25 & 0.01997 & 87213 & 0.0399744513 & 0.0000264005 & 0.00002 & 64024 \\
\hline 26 & 0.01921 & 18489 & 0.0384388223 & 0.0000176006 & 0.00001 & 76013 \\
\hline 27 & 0.01850 & 16206 & 0.0370167499 & $0.00001 \quad 17338$ & 0.00001 & 17341 \\
\hline 28 & 0.01784 & 19892 & 0.0356960933 & 0.0000078226 & 0.00000 & 78227 \\
\hline 29 & 0.01722 & 77380 & $0.03446 \quad 63822$ & 0.0000052151 & 0.00000 & 52151 \\
\hline 30 & 0.01665 & 43430 & 0.0333185392 & 0.0000034767 & 0.00000 & 34767 \\
\hline 31 & 0.01611 & 78618 & 0.0322446552 & $0.00000 \quad 23178$ & 0.00000 & 23178 \\
\hline 32 & 0.01561 & 48434 & 0.0312378079 & 0.0000015452 & 0.00000 & 15452 \\
\hline 33 & 0.01514 & 22533 & 0.0302919125 & 0.0000010301 & 0.00000 & 10301 \\
\hline 34 & 0.01469 & 74132 & 0.0294015987 & $0.00000 \quad 06868$ & 0.00000 & 06868 \\
\hline 35 & 0.01427 & 79499 & 0.0285621087 & $0.00000 \quad 04578$ & 0.00000 & 04578 \\
\hline 36 & 0.01388 & 17532 & 0.0277692127 & $0.00000 \quad 03052$ & 0.00000 & 03052 \\
\hline 37 & 0.01350 & 69405 & 0.0270191374 & 0.0000002035 & 0.00000 & 02035 \\
\hline 38 & 0.01315 & 18267 & 0.0263085061 & $0.00000 \quad 01357$ & 0.00000 & 01357 \\
\hline 39 & 0.01281 & 48994 & 0.0256342880 & 0.0000000904 & 0.00000 & 00904 \\
\hline 40 & 0.01249 & 47969 & 0.0249937549 & 0.0000000603 & 0.00000 & 00603 \\
\hline
\end{tabular}


TABLES $3 \& 4$

Values of $I_{k, 3}, I_{k, 3}^{*}$ and $I_{k,-1} / 2^{k+1}, I_{k,-1}^{*} / 2^{k+1}$

\begin{tabular}{|c|c|c|c|c|}
\hline k & $I_{k, 3}$ & ${ }_{k, 3}^{*}$ & $I_{k,-1} / 2^{k+1}$ & $I_{k,-1}^{*} / 2^{k+1}$ \\
\hline 1 & $0.15623 \quad 63163$ & - & 0.8281604155 & - \\
\hline 2 & 0.0461697930 & - & 0.8962281338 & - \\
\hline 3 & 0.0173267749 & 0.3202175927 & 0.9491826604 & 1.1546150481 \\
\hline 4 & $0.00701 \quad 00147$ & $0.04946 \quad 74154$ & 0.9772542460 & 1.0428005160 \\
\hline 5 & 0.0029145579 & 0.0118403416 & 0.9903967899 & 1.0141856891 \\
\hline 6 & $0.00122 \quad 19741$ & $0.00344 \quad 14251$ & 0.9961130213 & 1.0049808868 \\
\hline 7 & 0.0005123332 & 0.0011135709 & 0.9984756980 & 1.0017879993 \\
\hline 8 & 0.0002140033 & 0.0003848437 & 0.9994164949 & 1.0006469013 \\
\hline 9 & $0.00008 \quad 89236$ & 0.0001388317 & 0.9997807591 & 1.0002343666 \\
\hline 10 & 0.0000367442 & $0.00005 \quad 15626$ & 0.9999188141 & 1.0000847609 \\
\hline 11 & $0.00001 \quad 51021$ & 0.0000195430 & $0.99997^{\circ} 02797$ & 1.0000305571 \\
\hline 12 & $0.00000 \quad 61770$ & 0.0000075146 & 0.9999892195 & 1.0000109745 \\
\hline 13 & 0.0000025158 & 0.0000029197 & 0.9999961186 & 1.0000039260 \\
\hline 14 & $0.00000 \quad 10210$ & $0.00000 \quad 11430$ & 0.9999986111 & 1.0000013991 \\
\hline 15 & $0.00000 \quad 04131$ & $0.00000 \quad 04500$ & 0.9999995055 & 1.0000004968 \\
\hline 16 & $0.00000 \quad 01667$ & $0.00000 \quad 01779$ & 0.9999998247 & 1.0000001758 \\
\hline 17 & $0.00000 \quad 00672$ & $0.00000 \quad 00705$ & 0.9999999381 & 1.0000000620 \\
\hline 18 & $0.00000 \quad 00270$ & $0.00000 \quad 00280$ & 0.9999999782 & 1.0000000218 \\
\hline 19 & $0.00000 \quad 00109$ & $0.00000 \quad 00112$ & 0.9999999924 & $1.00000 \quad 00077$ \\
\hline 20 & 0.0000000044 & $0.00000 \quad 00044$ & 0.9999999973 & $1.00000 \quad 00027$ \\
\hline 21 & $0.00000 \quad 00017$ & $0.00000 \quad 00018$ & 0.9999999991 & 1.0000000009 \\
\hline 22 & 0.0000000007 & $0.00000 \quad 00007$ & 0.9999999997 & 1.0000000003 \\
\hline 23 & 0.0000000003 & 0.0000000003 & 0.9999999999 & 1.0000000001 \\
\hline 24 & 0.0000000001 & 0.0000000001 & 1.0000000000 & 1.0000000000 \\
\hline
\end{tabular}

The values of the integrals for $s=0$ and 2, or the four ordinary Howland integrals, are referred to the existing tables in the papers [3], [4], [5]. When the values of other integrals are needed, they can be found from the known values by using the recurrence relations in (2) without losing accuracy.

The values of $I_{k,-1}$ and $I_{k,-1}^{*}$ may be checked by the recurrence relations in (2). Those of the other integrals may be checked by the relations shown below. They are, for $s \geqslant 1$,

$$
\begin{array}{ll}
\sum_{k=0}^{\infty} I_{2 k+1, s}=\frac{1}{s}-I_{1, s}, & \sum_{k=1}^{\infty} k I_{2 k, s}=\frac{1}{s^{2}}-I_{2, s}, \\
\sum_{k=1}^{\infty} I_{2 k+1, s}^{*}=\frac{1}{s}, & \sum_{k=2}^{\infty} k I_{2 k, s}^{*}=\frac{1}{s^{2}},
\end{array}
$$

and for $I_{1, s}$ and $I_{3, s}^{*}$,

$$
\begin{array}{ll}
\sum_{s=0}^{\infty}(-1)^{s} I_{1,2 s+1}=\frac{\pi}{4}-V_{0}, & \sum_{s=1}^{\infty}(-1)^{s+1} I_{1,2 s}=\frac{1}{2} I_{1,0}-\frac{1}{2} I I I_{1}, \\
\sum_{s=0}^{\infty}(-1)^{s} I_{3,2 s+1}^{*}=\frac{4}{3} V_{2}^{*}-\frac{\pi^{3}}{24}, & \sum_{s=1}^{\infty}(-1)^{s+1} I_{3,2 s}^{*}=\frac{1}{2} I_{3,0}^{*}-\frac{1}{2} I I I_{3}^{*},
\end{array}
$$


where

$$
\begin{gathered}
V_{0}=\int_{0}^{\infty} \frac{\sinh x d x}{\sinh 2 x+2 x}=0.5268563984 \\
I I I_{1}=2 \int_{0}^{\infty} \frac{x \tanh x d x}{\sinh 2 x+2 x}=0.4744296568 \\
V_{2}^{*}=\frac{1}{2} \int_{0}^{\infty} \frac{x^{2} \sinh x d x}{\sinh 2 x-2 x}=1.4087956089 \\
I I_{3}^{*}=\frac{4}{3} \int_{0}^{\infty} \frac{x^{3} \tanh x d x}{\sinh 2 x-2 x}=1.4150633610 .
\end{gathered}
$$

Owing to slow convergence, the tail part of the series in (22) can be found with the aid of the Euler transformation [1] or from the values of the series of inverse powers of natural numbers. The evaluation of the four integrals in (23) was considered by the author in a previous paper [2], but the values were given to $6 \mathrm{D}$ only. It is straightforward to evaluate again the first three integrals. It may be more convenient to evaluate the last one from the following series:

$$
I I I_{3}^{*}=\frac{1}{3} \sum_{n=1}^{\infty}\left(\begin{array}{c}
2 n+2 \\
2
\end{array}\right)\left(I_{2 n+2,0}^{*}-U_{2 n+3}\right),
$$

where

$$
U_{n}=1+\frac{1}{3^{n}}+\frac{1}{5^{n}}+\frac{1}{7^{n}}+\cdots, \quad(n \geqslant 2) .
$$

Department of Mathematics

Virginia Polytechnic Institute and State University

Blacksburg, Virginia 24061

1. T. J. I'A. Bromwich, Theory of Infinite Series, 2nd ed., Macmillan, New York, 1926, p. 62.

2. C. B. LING, "Tables of values of 16 integrals of algebraic-hyperbolic type," MTAC, v. 11, 1957, pp. $160-166$.

3. C. B. LiNG \& J. LiN, “A new method of evaluation of Howland integrals,” Math. Comp., v. 25, 1971, pp. $331-337$.

4. C. B. Ling, "Further evaluation of Howland integrals," Math. Comp., v. 32, 1978, pp. 900-904.

5. C. B. LiNG \& M. J. WU, "Evaluation of integrals of Howland type involving a Bessel function," Math. Comp., v. 38, 1982, pp. 215-222. 